\title{
An Extensive Method for Maintenance of Sterility in Mammalian Cell Culture Laboratory Routine
}

\author{
Selcen Çelik-Uzuner* (i) and Uğur Uzuner \\ Department of Molecular Biology and Genetics, Faculty of Science, Karadeniz Technical University, \\ Trabzon 61080, Turkey; uguruzuner@ktu.edu.tr \\ * Correspondence: selcen.celik@ktu.edu.tr
}

Received: 14 September 2017; Accepted: 27 October 2017; Published: 31 October 2017

\begin{abstract}
Cell culture laboratories are designed to secure sterile environments for biological studies, and its susceptibility to biological contaminants is a major problem for researchers. Contamination can generally be overcome by frequently disposing of contaminated materials, cleaning and maintaining the sanitation of working areas, but in some cases, it is hard to eradicate the source(s) of contaminating agents completely. In particular, mold and fungal-based contaminants could be a devastating problem for any laboratory. Therefore, various contamination types can repeat over time and result in an increasing problem in the lab. To control and eliminate possible contaminations, a periodic cleaning treatment with disinfectant materials should be a routine procedure of every specialized laboratory, regardless of the existence of any contamination. The conventional method for maintaining hygiene in cell culture laboratories is to clean all surfaces with $70 \%$ ethanol; however, this may not be the complete solution. This paper presents a short and easy way to check whether any contamination source(s) are present in laboratories. After identification of the most contaminant-rich areas, without any distinction, each laboratory surface where the culturing samples were taken was comprehensively treated with suggested concentrations of ethanol, sodium hypochlorite and Virkon-S solutions. The method might be useful to regularly check and maintain the cleanliness and hygiene of entire cell culture laboratories to enable the control of possible contaminations in advance.
\end{abstract}

Keywords: cell culture; laboratory; contamination; sterility; aseptic techniques

\section{Introduction}

Cell culture applications are widely performed in specified cell culture laboratories to study numerous aspects of molecular biology, including cellular metabolism, therapy, genetics and biotechnology. These laboratories are designated as isolated areas with good ventilation and equipped with specified instruments providing sterile environments in appropriate locations, and are desired to meet the regulations for biological safety requirements. Laboratories used for biological research are classified in terms of biosafety levels (BSL) which represent the content of laboratory work regarding the organisms' and /or agents' possible effects on laboratory workers [1]. For instance, organisms which do not cause any disease are experienced at the lowest level of those laboratories (BSL1), and organisms which cause transmitted diseases such yellow fever are experienced at the highest level of laboratories (BSL-3). BSL2 laboratories are designated for the use of some agents and organisms that can cause moderate hazard [1]. Cell culture applications are performed in BSL2 laboratories and these are rather more expensive than that of BSL1 laboratories and also require sterile and disposable consumables and plastic-wares to maintain sterility during experimental procedures. Precautions should be taken depending on the biosafety level of laboratories. In BSL2 laboratories, sterility is usually secured by the common use of ethanol for regular cleaning purposes in the working areas and spraying it onto the lids and edges of almost all laboratory materials before each use [2,3]. This standard application 
for the maintenance of laboratory sterility can also be enhanced by additional precautions including the addition of various, appropriate and effective antibiotics to the cell culture media. However, many researchers do not prefer to use antibiotics in culture media since antibiotics may interfere with the experimental outcome in some cell lines $[4,5]$.

Although strict rules are applied in cell culture laboratories, there can be still some challenges to maintain the sterility of working areas and materials. Biological contamination that is defined as the existence of unwanted living beings, i.e., bacteria, fungi or yeast, in cultures is a major problem in such specialized laboratories. These can not only interfere with the metabolism of cells in culture but also induce cytotoxicity. The contamination risk can intensify in particular when unskilled staff and/or too many researchers work at the same time in a laboratory, resulting in the loss of sterility control. Contamination is dangerous due to its potential to be spread to many areas, cell lines and materials; and could be quite costly if this cannot be prevented or eradicated quickly.

The contamination whose source(s) cannot be found is the most important difficulty for laboratory work. Although general cleaning with ethanol is the common way to control sterility in laboratories, we suggest that this cannot be a precise method to avoid contamination possibilities. In our laboratory, we have designed and used a routine method to check whether all working areas are appropriately clean and without any contamination, and sterilize contaminated areas by a triple cleaning strategy. To do this, the extensive sanitation method included culturing the samples from various laboratory areas (incubator, laminar flow cabinet, microscope, liquid nitrogen tank, centrifuge and water bath) and consumables (culture media, liquid nitrogen, water) on blood agar plates for two days. It was identified that incubator was predominantly the most problematic area. Interestingly, the microscope was defined as another source for contaminants. After identification of the most contaminant-rich areas, without any distinction, each laboratory surface where the culturing samples were taken was comprehensively treated with suggested concentrations of ethanol, sodium hypochlorite and Virkon-S solutions. This experience presents a model of extensive, cost-effective and rapid evaluation to maintain sterility in any conventional cell culture laboratory.

\section{Results}

During diverse cell culture applications, one of the flasks was identified with an unknown bacterial contamination. Examples of clean and contaminated cultures are illustrated in Figure 1. Possible contamination sources derived from various laboratory instruments and / or any surfaces were then examined for the major contaminant origins. For this, numerous samples from different areas including (1) water bath, (2) incubator shelves, (3) water tray and (4) internal filter of incubator, (5) working surface and (6) internal window surfaces of laminar flow cabinet, (7) centrifuge rotors, (8) liquid nitrogen, (9) internal surface of the liquid nitrogen tank lid, (10) lid surroundings of cell line cryovials and (11) main sampling stage and objectives of fluorescence microscope were taken using sterile cotton swabs, after that, each sample was rubbed onto sterile blood agar plates. The samples from sterile culture media did not show any bacterial colony growth, but the sample from the contaminated culture revealed bacterial growth, as expected (Figure 2A,B). Several plates that did not have any inoculum from the contaminated culture were incubated in a different laboratory under the same treatment conditions as control (Figure 2C). In order to determine if the current contamination stemmed from the incubator, two of these control plates were independently incubated in the incubators (one in the different laboratory with no contamination and one is available within the contaminated lab), one without any plate coverage (plate lid was intentionally removed) and one with closed plate lid (usual incubation of a culture plate). Although neither control group had any inoculums from the contaminated culture or sampling from various sources (Figure 2C), only the plate from the contaminated laboratory was left with its lid off and located away from internal filter in the incubator revealed the bacterial colony formation (Figure 2D). However, leaving the plate lid open did not make any difference (no bacterial or fungal growth identified) for the control plate incubated in the non-contaminated lab. Another lid-off control plate placed next to the internal filter of the incubator 
also revealed a small colony formation (Figure 2E). In contrast, the control plate incubated as lid on at the same incubator location was clear (Figure 2F). This could suggest that air within the incubator was most likely the source of contamination. It is not only the airflow of the incubator, but also from the incubator's shelf which resulted in a big colony development through the same incubation period (Figure 2G). Interestingly, the microscope stage and objectives were also identified as the contaminated surfaces (Figure 2H). The rest of plates with samples from the water tray of incubator, liquid nitrogen itself, internal side of liquid nitrogen tank lid, water bath, laminar flow areas, outside surfaces of cryovials of different cell stocks and centrifuge rotors were free of any colony formation or bacterial growth (data not shown).

A

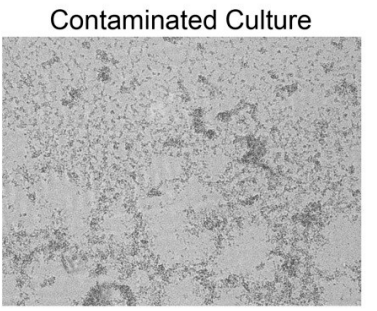

B

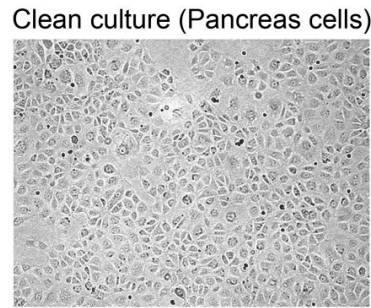

C

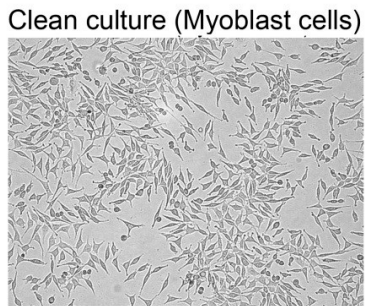

Figure 1. Representative microscopy images of cells. (A) shows a contaminated culture, $(\mathbf{B}, \mathbf{C})$ show clean and appropriate cell culture for pancreas and myoblast cells, respectively. Images were captured using $10 \times$ magnification by Zeiss Axiovert A1.

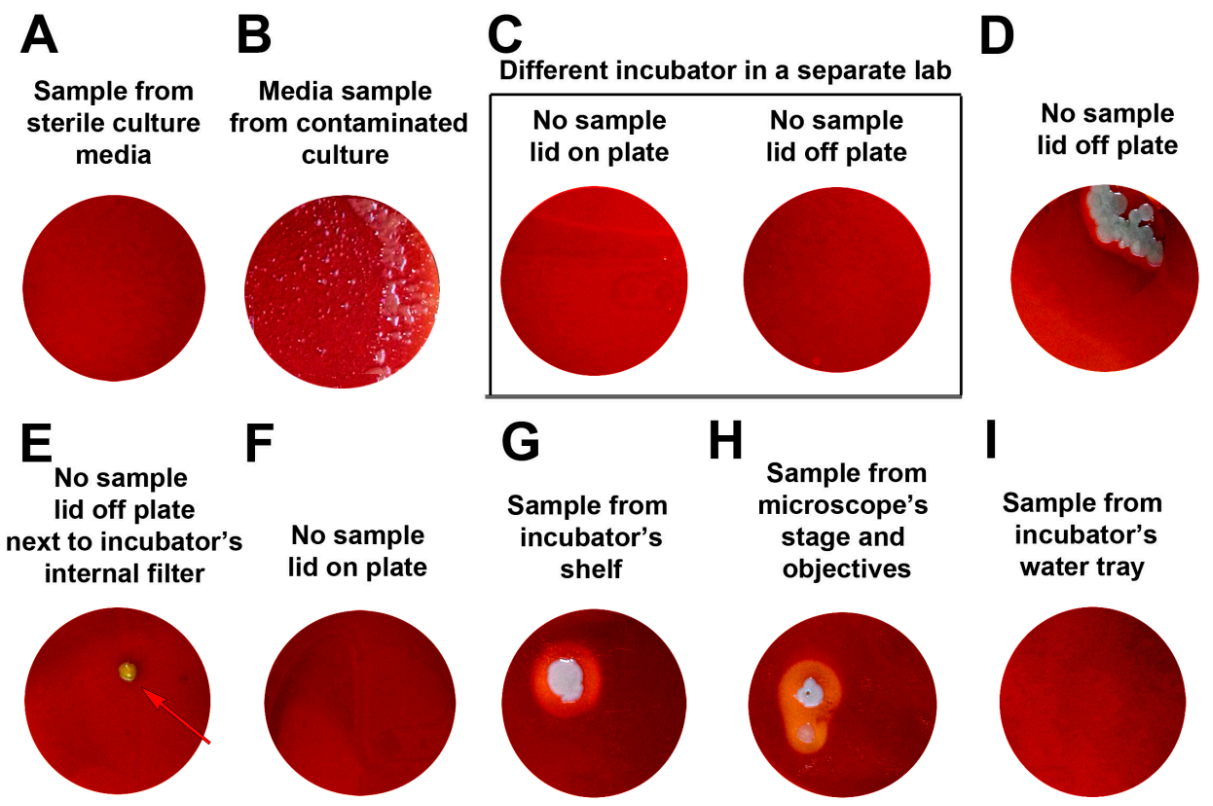

Figure 2. Representative blood agar plates with cultured samples from different sources of the laboratory. (A,B) show media control plates including negative (clean media) and positive control (contaminated media) samples, respectively. (C) shows control plates with or without lids incubated in a different laboratory providing counterparts of (D-F). (G-I) show cultures from the shelves of the incubator, microscope stage/objectives and the water tray of the incubator, respectively.

After figuring out the contamination sources, a triple cleaning strategy including $70 \%$ ethanol, $1 \%$ sodium hypochlorite and $1 \%$ Virkon-S solution was applied to contaminated areas and all other surfaces except for microscopy parts. All were treated with ethanol spraying once again. The fluorescence microscope stage was cleaned using $70 \%$ ethanol alone and the objectives were treated using a mixed solution of $70 \%$ ether (100\%) and 30\% ethanol (100\%). The incubator was also sterilized 
using a mobile UV lamp for $15 \mathrm{~min}$ followed by the incubation with a disinfection kit (provided by the manufacturer). This kit (NüveDis ${ }^{\mathrm{TM}}$ ) is in a powder form including copper sulphate and ethidium bromide and this content was dissolved into sterile distilled water in the water tray of the incubator at $90{ }^{\circ} \mathrm{C}$ for $9 \mathrm{~h}$ according to the manufacturer's instructions. Following this comprehensive cleaning process, the same contamination screening procedure and related experimental steps described above were surveyed once again and no colony formation was identified for the related samples obtained from those surfaces (data not shown).

\section{Discussion}

The experimental design presented here serves as a rapid evaluation process of possible contamination source(s) in the mammalian cell culture laboratories. Through testing the numerous laboratory equipment and their special accessories, we identified that the major source of contamination was the incubator. Incubators are one of the main instruments in cell culture laboratories. These instruments are quite sensitive to the degree of internal air flow and can be manipulated by the adverse effects of outside air. The circulating air within the instrument thus must be isolated from the direct flow of laboratory air conditioning and their interaction should be diminished as much as possible so that the contamination risk could be minimized. Therefore, incubators should not be located near to air conditioning blowers. Moreover, the filters for air conditioning machines should be equipped with convenient accessories. Silver ion filters and antimicrobial filters suitable for air conditioning machines might thus be the first hand efficient barrier against the air-born external contamination agents for cell culture laboratories. Air conditioning filters which can capture most of the bacterial and fungal contamination sources internally should also be changed regularly to eliminate any leak to the inside of laboratories.

Leaving incubator lids open longer than the sufficient time during the laboratory processes may become another possible cause for contamination of internal air. To avoid this, researchers should open and close the doors of incubators with care and keep the processing time as short as possible. The inside of door and shelves should be sprayed with ethanol regularly. Water trays placed on the base of incubators should be also considered for regular cleaning surveys. Double distilled water $\left(\mathrm{ddH}_{2} \mathrm{O}\right.$ ) should be preferred for all cell culture laboratory routines and $\mathrm{ddH}_{2} \mathrm{O}$ stocks must always be used and refreshed once a week. Filters of the incubator should be also changed regularly to make sure that filters are working appropriately. Disinfection with kits or UV lamp can be a useful precaution if possible.

From the current survey, it was unexpectedly identified that fluorescence microscope parts were also contaminated. However, in theory, all areas are susceptible to contamination. Regular cleaning of all instruments and/or surfaces is therefore crucial. The common disinfectant used in laboratories is ethanol, but proper cleaning sometimes needs the use of additive disinfectants such as Virkon-S solution which is an oxidizing agent. The active substance of Virkon-S solution is potassium peroxymonosulfate $(50 \%)$ and it also includes sodium chloride. Potassium peroxymonosulfate is used to decontaminate not only chemical compounds, but also microorganisms [6]. This chemical has essential effects on a wide range of microorganisms including viruses [7,8] and bacteria [9]. However, attention is required during its common use since this chemical can be corrosive for some surfaces.

Blood agar has been commonly used to detect any colonies that can live in mammalian cell culture sources and/or on accessories. However, liquid growth media can alternatively be used for the culturing any contaminants for growth as most contaminants can grow well within liquid culture media.

The sources of contamination have been completely eradicated by the method described above; yet carrying out further investigations on the identified of pollutants is the researchers' choice. 


\section{Materials and Methods}

\subsection{Cells}

Mammalian cells used were (i) primary embryonic fibroblasts (mouse), (ii) NIH-3T3 cells (ATCC ${ }^{\circledR}$ CRL-1658 ${ }^{\mathrm{TM}}$ ), (iii) myoblast cells (Sol8, ATCC ${ }^{\circledR}$ CRL-2174 ${ }^{\mathrm{TM}}$ ), and (iv) pancreatic cancer cells (AR42J, ECACC 93100618). Cells (i,ii) were cultured in DMEM (Capricorn, Cat No. 319-007-CL), media including 10\% fetal bovine serum (FBS) (Gibco, Cat No. 10270) or bovine calf serum (Sigma, 12133C) respectively, and 1\% Penicillin-streptomycin (Wisent, Cat No. 450201). Primary fibroblast culture also included 1\% MEM-NEAA (Capricorn, Cat No. NEAA-B). Myoblast cells (iii) were cultured in DMEM media including 20\% FBS and antibiotics. Pancreatic cancer cells were cultured in RPMI (Sigma, R8758) media including 10\% FBS and antibiotics. All media was filtered by 0.22 micron filters (Aisimo, ASCA2233), and all cells were cultured at $37^{\circ} \mathrm{C}$ with humidified air by $5 \% \mathrm{CO}_{2}$. All cells were cultured within sterile flasks with vented lids.

\subsection{Blood Agar Plate Preparations}

Agar powder used was obtained from BioMerieux, France. To prepare blood agar plates, $45 \mathrm{~mL}$ agar-agar $(15 \mathrm{~g} / \mathrm{L})$ was autoclaved at $121{ }^{\circ} \mathrm{C}$ and $3 \mathrm{~mL}$ defibrinated sheep blood $(6.25 \%$ final concentration) was added to cooled agar solution. $4 \mathrm{~mL}$ of this mixture was poured to each $60 \mathrm{~mm}$ sterile petri dish. Samples from surfaces were taken using sterile cotton swabs, and spread on blood agar dishes.

\section{Conclusions}

This study suggests a deeper cleaning strategy for general laboratory use than the standard cleaning with only ethanol, and proposes an easy and applicable method for cell culture researchers to control possible contaminations in the laboratory. Maintenance of sterility is worthwhile since molecular biology studies are highly expensive and involve much time and effort.

Acknowledgments: Authors thank to Elif Ayazoglu Demir for the preparations of experiments.

Author Contributions: Selcen Çelik-Uzuner performed cell culture, Ugur Uzuner performed agar cultures. Selcen Çelik-Uzuner and Ugur U̧zuner wrote the manuscript.

Conflicts of Interest: The authors declare no conflict of interest.

\section{References}

1. Richmond, J.Y.; McKinney, R.W. Biosafety in Microbiological and Biomedical Laboratories, 5th ed.; U.S. Department of Health and Human ServicesPublic Health Service, Public Health Service Centers for Disease Control and Prevention, National Institutes of Health: Atlanta, GA, USA, 2009.

2. Bykowski, T.; Stevenson, B. Aseptic Technique. Curr. Protoc. Microbiol. 2008. [CrossRef]

3. Cote, R.J. Aseptic Technique for Cell Culture. In Current Protocols in Cell Biology; John Wiley \& Sons, Inc.: Franklin, NJ, USA, 2001; Chapter 1, Unit 1.3.

4. Cohen, S.; Samadikuchaksaraei, A.; Polak, J.M.; Bishop, A.E. Antibiotics Reduce the Growth Rate and Differentiation of Embryonic Stem Cell Cultures. Tissue Eng. 2006, 12, 2025-2030. [CrossRef] [PubMed]

5. Ryu, A.H.; Eckalbar, W.L.; Kreimer, A.; Yosef, N.; Ahituv, N. Use Antibiotics in Cell Culture with Caution: Genome-Wide Identification of Antibiotic-Induced Changes in Gene Expression and Regulation. Sci. Rep. 2017, 7. [CrossRef] [PubMed]

6. Anipsitakis, G.P.; Tufano, T.P.; Dionysiou, D.D. Chemical and Microbial Decontamination of Pool Water Using Activated Potassium Peroxymonosulfate. Water Res. 2008, 42, 2899-2910. [CrossRef] [PubMed]

7. Su, X.W.; D'Souza, D.H. Inactivation of Human Norovirus Surrogates by Benzalkonium Chloride, Potassium Peroxymonosulfate, Tannic Acid, and Gallic Acid. Foodborne Pathog. Dis. 2012, 9, 829-834. [CrossRef] [PubMed] 
8. Jang, Y.; Lee, J.; So, B.; Lee, K.; Yun, S.; Lee, M.; Choe, N. Evaluation of Changes Induced by Temperature, Contact Time, and Surface in the Efficacies of Disinfectants against Avian Influenza Virus. Poult. Sci. 2014, 93, 70-76. [CrossRef] [PubMed]

9. Mainous, M.E.; Smith, S.A. Efficacy of Common Disinfectants against Mycobacterium Marinum. J. Aquat. Anim. Health 2005, 17, 284-288. [CrossRef] 